\title{
Multispectral imaging system based on light-emitting diodes for the detection of melanomas and basal cell carcinomas: a pilot study
}

\author{
Xana Delpueyo \\ Meritxell Vilaseca \\ Santiago Royo \\ Miguel Ares \\ Laura Rey-Barroso \\ Ferran Sanabria \\ Susana Puig \\ Josep Malvehy \\ Giovanni Pellacani \\ Fernando Noguero \\ Giuseppe Solomita \\ Thierry Bosch
}




\title{
Multispectral imaging system based on light-emitting diodes for the detection of melanomas and basal cell carcinomas: a pilot study
}

\author{
Xana Delpueyo, ${ }^{\mathrm{a}, \star}$ Meritxell Vilaseca, ${ }^{\mathrm{a}}$ Santiago Royo, ${ }^{\mathrm{a}}$ Miguel Ares, ${ }^{\mathrm{a}}$ Laura Rey-Barroso, ${ }^{\mathrm{a}}$ Ferran Sanabria, ${ }^{\mathrm{a}}$ \\ Susana Puig, ${ }^{b}$ Josep Malvehy, ${ }^{b}$ Giovanni Pellacani, ${ }^{c}$ Fernando Noguero, ${ }^{d}$ Giuseppe Solomita, ${ }^{e}$ and \\ Thierry Bosch \\ ${ }^{a}$ Technical University of Catalonia, Centre for Sensors, Instruments and Systems Development (CD6), Terrassa, Spain

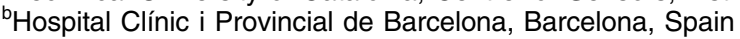 \\ 'Università di Modena e Reggio Emilia, Modena, Italy \\ ${ }^{\mathrm{d}}$ Carril Instruments S.L., Barcelona, Spain \\ eMavig GmbH, Munich, Germany \\ Institut National Polytechnique de Tolouse, Toulouse, France
}

\begin{abstract}
This article proposes a multispectral system that uses the analysis of the spatial distribution of color and spectral features to improve the detection of skin cancer lesions, specifically melanomas and basal cell carcinomas. The system consists of a digital camera and light-emitting diodes of eight different wavelengths $(414$ to $995 \mathrm{~nm})$. The parameters based on spectral features of the lesions such as reflectance and color, as well as others empirically computed using reflectance values, were calculated pixel-by-pixel from the images obtained. Statistical descriptors were calculated for every segmented lesion [mean $(\tilde{x})$, standard deviation $(\sigma)$, minimum, and maximum]; descriptors based on the first-order statistics of the histogram [entropy $(E p)$, energy $(E n)$, and third central moment $\left.\left(\mu_{3}\right)\right]$ were also obtained. The study analyzed 429 pigmented and nonpigmented lesions: 290 nevi and 139 malignant (95 melanomas and 44 basal cell carcinomas), which were split into training and validation sets. Fifteen parameters were found to provide the best sensitivity $(87.2 \%$ melanomas and $100 \%$ basal cell carcinomas) and specificity (54.5\%). The results suggest that the extraction of textural information can contribute to the diagnosis of melanomas and basal cell carcinomas as a supporting tool to dermoscopy and confocal microscopy. ๑ 2017 Society of Photo-Optical Instrumentation Engineers (SPIE) [DOI: 10.1117/1.JBO.22.6.065006]
\end{abstract}

Keywords: skin cancer; melanoma; basal cell carcinoma; multispectral; light-emitting diodes.

Paper 170088RR received Feb. 9, 2017; accepted for publication Jun. 13, 2017; published online Jun. 29, 2017; corrected Jul. 20, 2017.

\section{Introduction}

Globally, one in three cancers is a cancer of the skin. In Europe, the United States, and Australia, the incidence of skin cancer is increasing rapidly. Melanoma, which represents $4 \%$ of all skin cancer lesions, is the most aggressive and lethal of all skin cancer forms. ${ }^{1}$ Approximately $90 \%$ of skin cancers are caused by ultraviolet (UV) light from daylight or tanning booths. The World Health Organization estimates that 60,000 people die every year from excess solar UV radiation: 48,000 from melanoma and 12,000 from carcinomas. Crucially, early detection and treatment significantly increases the 5-year survival rate.

Currently, the technique most widely used by dermatologists for the detection of skin cancer is visual inspection through a dermoscope, a handheld device with a magnifying lens and a white and uniform illumination field. The light is often polarized to remove specular reflection from the skin surface to capture information from deeper tissue layers. Dermoscopy allows the identification of different structures, patterns, and colors in skin lesions characteristic of malignant (melanoma and basal cell carcinoma) and benign lesions (seborrheic keratosis, hemangioma, lipoma, and wart). Histological examination, which requires the surgical excision of the tumor and is the clinical gold standard, provides diagnostic confirmation. A limitation of

*Address all correspondence to: Xana Delpueyo, E-mail: xana.delpueyo@upc .edu dermoscopy is that it produces a large number of false positives, thus contributing to the high direct annual costs for the diagnosis and treatment of skin cancer. ${ }^{2}$

Color and spectral imaging technology that enhance and analyze spectral properties of the skin are currently being explored to improve early detection and diagnosis of skin cancer. Spectral properties are caused by chromophores such as melanin, hemoglobin, and water, which differ among skin lesions of different etiologies.

In addition to the commercial devices, SIAscope, ${ }^{3}$ MelaFind, ${ }^{4}$ and prototypes such as those developed by Bekina et al. ${ }^{5}$ and Kapsokalivas et al. ${ }^{6}$ have already been proposed as tools for improving skin cancer diagnosis. Most devices only use three spectral bands in the visible range (typically three-color RGB channels), which limits their spectral resolution, and an additional one located at the near-infrared (NIR) range. Notably, most tools only analyze the averaged color and spectral properties.

This study presents a new handheld multispectral system with spectral bands along the visible (VIS) and the NIR ranges for the diagnosis of skin cancer that takes also into account the distribution of color and spectral features of the skin lesion, i.e., it includes texture or spatial information instead of just the corresponding averaged values. We show the methodology performed to setup and characterize the whole system, including

$1083-3668 / 2017 / \$ 25.00$ (c) 2017 SPIE 

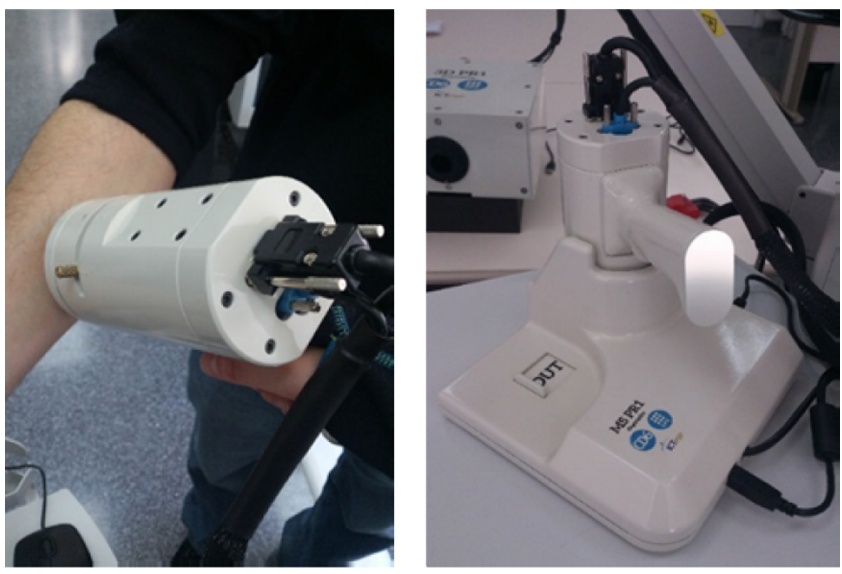

Fig. 1 Different views of the multispectral system developed.

the protocol followed to select the most suitable color and spectral parameters to detect skin cancer lesions, specifically melanomas and basal cell carcinomas, and to differentiate them from nevi. The results of spectral and spatial properties corresponding to real lesions analyzed at a clinical site are also presented.

This study participates in the European Project DIAGNOPTICS "diagnosis of skin cancer using optics" (ICT PSP seventh call for proposals 2013), with the objective to develop a multiphotonic diagnostic platform including multispectral and 3-D techniques, ${ }^{7}$ blood flow analysis based on self-mixing, ${ }^{8}$ and confocal microscopy ${ }^{9}$ for in vivo imaging of skin cancer lesions. The aim of these new technologies is to achieve a higher detection ratio and better prognostic evaluation of skin cancer at earlier stages when compared with current methods.

\section{Material and Methods}

\subsection{Equipment}

Figure 1 shows the multispectral system developed; it consists of a handheld ergonomic cylinder of $\sim 10 \mathrm{~cm}$ in length, $7.5 \mathrm{~cm}$ in diameter, and a weight of $0.5 \mathrm{Kg}$. The cylinder contains a CCD monochrome camera (Sony ICX445ALA with $1280 \times 960$ pixels of sensitive area and 12-bit depth of digitalization) and a lens (Cinegon 1.8/16-0901) that can record skin lesions focused at $4 \mathrm{~cm}$ with $15 \times 20 \mathrm{~mm}$ field of view; moreover, a set of 32 light-emitting diodes (LED) of eight different wavelengths (414, 447, 477, 524, 671, 735, 890, and $995 \mathrm{~nm})$ was chosen in accordance with the absorption curves of the principal chromophores of the skin, especially taking into account their most representative minimums and maximums and the spectral bands with considerable differences among them allowing characterization of the tissue constituents. Commercial availability of LEDs in the analyzed range (400 to $1000 \mathrm{~nm}$ ) was also considered as a limiting factor. Table 1 contains information of the relationship between the peaks of the LEDs chosen and the reason for selection.

In addition, the system contains two rotating polarizers located in front of the LEDs and the lens, respectively, which allow the removal of the specular reflection from the skin if desired.

Figure 2 shows the spectral emission of the LEDs included in the ring of the multispectral system. Measurements were performed with a commercial scanning spectrometer model Spectro 320 R5 of Instrument Systems. As can be seen, their full width
Table 1 Reason of wavelengths selection.

\begin{tabular}{ll} 
Wavelength $(\mathrm{nm})$ & \multicolumn{1}{c}{ Reason of selection } \\
\hline 414 & $\begin{array}{l}\mathrm{Hb}^{\mathrm{a}} \text { and } \mathrm{HbO}_{2}{ }^{\mathrm{b}} \text { peaks of maximum absorption } \\
\text { Melanin maximum absorption }\end{array}$ \\
447 & Bilirubin peak of maximum absorption \\
477 & $\mathrm{Hb}^{\mathrm{a}}$ minimum absorption \\
524 & $\begin{array}{l}\text { Intermediate wavelength allowing a complete } \\
\text { spectral sampling }\end{array}$ \\
671 & $\mathrm{HbO}_{2}{ }^{\mathrm{b}}$ minimum absorption \\
735 & $\begin{array}{l}\text { Typical wavelength used in pulse oximetry } \\
\left(\text { allowing differentiation between } \mathrm{Hb}^{\mathrm{a}} \text { and } \mathrm{HbO}_{2}{ }^{\mathrm{b}}\right)\end{array}$ \\
890 & $\begin{array}{l}\text { Typical wavelength used in pulse oximetry } \\
\text { (allowing differentiation between } \mathrm{Hb}^{\mathrm{a}} \text { and } \mathrm{HbO}_{2}{ }^{\mathrm{b}} \text { ) }\end{array}$ \\
& $\begin{array}{l}\text { Information from deeper layers of the skin } \\
995\end{array}$ \\
\hline
\end{tabular}

${ }^{\mathrm{a}} \mathrm{Hb}$, deoxyhemoglobin.

${ }^{\mathrm{b}} \mathrm{HbO}_{2}$, oxyhemoglobin.

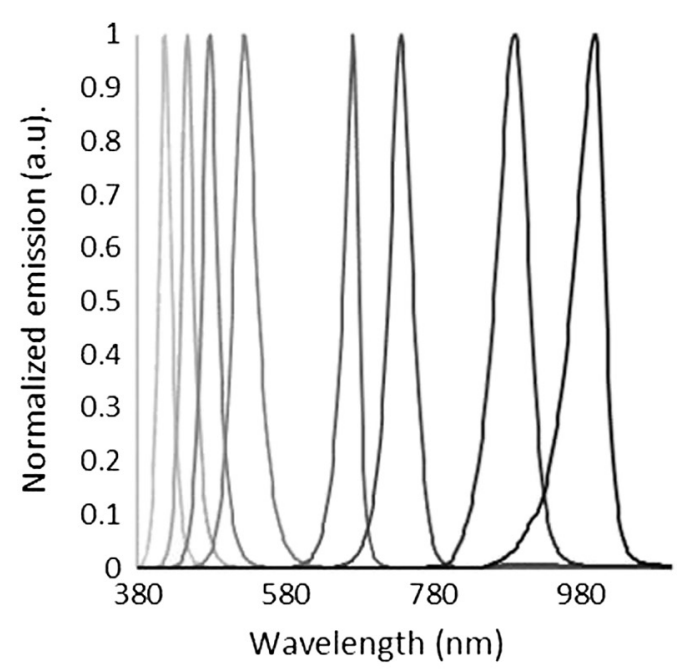

(a)

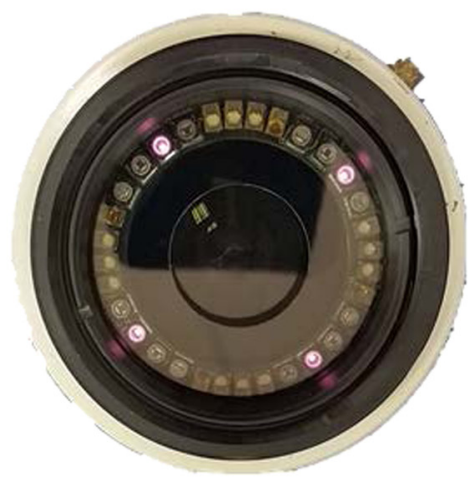

(b)

Fig. 2 LEDs included in the ring of the multispectral system: (a) spectral emission and (b) front picture of the ring. 
at half-maximum ranged from 15 to $40 \mathrm{~nm}$, which was considered enough to analyze the spectral features of lesions due to the fact that they are linked to rather smooth absorption curves as other authors have previously reported. ${ }^{10-12}$ The four LED units for each wavelength are distributed over a ring with an angular separation of $90 \mathrm{deg}$, generating a uniform and diffuse illumination on the skin.

To facilitate its use, the clinicians can place the head of the system on a base between measurements. In addition to its storing function (the power supply and electronic boards are inside), the main function of the base concerns calibration. To this end, the base incorporates a reference sample (Neutral 6.5 from XRite ColorChecker ${ }^{\circledR}$ Classic CCCR) used in the daily calibration of the system, which is carried out before starting measurements.

\subsection{Collection of the VIS-NIR Spectral Images and Parameters Analyzed}

Spectral images for the eight wavelengths (or spectral bands) available were obtained through an automated and sequential process of acquisition. A complete acquisition for all wavelengths lasted about $40 \mathrm{~s}$. Each lesion was only measured once as the repeatability achieved with the system was very good: a percentage of variation (standard deviation/mean $\times 100$ ) of less than $2.5 \%$ was registered for all wavelengths when 15 different acquisitions were made for 3 consecutive days ( 5 acquisitions per day).

Next, eight reflectance and eight absorbance images were computed considering the spectral images of the aforementioned reference sample as well as dark current images, taken without ambient light and for any skin sample placed in front of the system; the reflectance and absorbance at each pixel $(i, j)$ for a given wavelength $\lambda_{i}$ were then calculated as follows:

$$
\operatorname{Refl}_{\lambda n}(i, j)=\operatorname{Refl}_{\operatorname{ref}_{\lambda n}} \cdot \frac{\mathrm{DL}_{\lambda n}(i, j)-\mathrm{DL}_{0_{\lambda n}}(i, j)}{\operatorname{DL}_{\mathrm{ref} \lambda n}(i, j)-\mathrm{DL}_{0_{\lambda n}}(i, j)},
$$

$\operatorname{Abs}_{\lambda n}(i, j)=-\log \left[\operatorname{Refl}_{\lambda n}(i, j)\right]$

where $\operatorname{Refl}_{\lambda n}(i, j)$ is the reflectance, $\mathrm{DL}_{\lambda n}(i, j)$ is the digital level of the acquired raw image of the lesion, $\operatorname{DL}_{0_{\lambda n}}(i, j)$ is the digital level of the current dark image, $\mathrm{DL}_{\text {ref }}(i, j)$ is the digital level of the reference, i.e., the calibrated sample, $\operatorname{Refl}_{\operatorname{ref}_{\lambda n}}$ is the calibrated reflectance of the reference provided by the manufacturer, and $\operatorname{Abs}_{\lambda n}(i, j)$ is the absorbance. All images were acquired with the same offset, gain, and exposure time camera parameters as those used for the analyzed lesion.

Three different groups of parameters were calculated from the reflectance images of a lesion. The first group consisted of the pixel-by-pixel spline interpolation values (from 415 to $995 \mathrm{~nm}$ with steps of $10 \mathrm{~nm}$ ) of the eight previous reflectance and absorbance images to obtain more accurate information. Additionally, differences between normal skin and the lesion were also computed in reflectance and absorbance terms to prevent the patient's skin from influencing the results.

Additional images containing a second group of parameters were computed to look for color features of skin lesions; color coordinates of standard color representation spaces were used, such as those of the CIELAB color space ${ }^{13}$ in which color is represented with lightness $(L *)$, red-green $(a *)$, and yellowblue coordinates $(b *)$; alternatively; chroma $\left(C_{\mathrm{ab}}^{*}\right)$ and the hue angle can also be used $\left(h_{\mathrm{ab}}\right)$. More complex parameters based on CIELAB color coordinates were also considered such as color differences $(\Delta E)$ between each pixel of the lesion and the averaged color of the whole lesion and between each pixel of the lesion and the averaged color of the surrounding healthy skin or the individual typology angle (ITA), ${ }^{14}$ which is related with the hue angle and is commonly used to classify different kinds of skin.

The interpolated reflectance values (see above), illuminant CIE D65 and CIE 2 deg standard observer were used to compute the color data in all cases. ${ }^{15,16}$

The third group consisted of what we considered "empirical parameters," i.e., parameters empirically computed by operating with reflectance values at different wavelengths to enhance any particular spectral feature potentially different in healthy and malignant tissues that might be useful in discriminating among different types of skin lesions

$\operatorname{Par}_{m}(i, j)=f\left[\operatorname{Refl}_{\lambda n}(i, j)\right]$,

where $\operatorname{Par}_{m}(i, j)$ is a particular parameter and $f\left[\operatorname{Refl}_{\lambda n}(i, j)\right]$ is a function of the reflectance images computed from several wavelengths (they can be added, subtracted, multiplied, etc.) to highlight subtle differences among lesions of different etiologies. Various authors have suggested that some of these parameters can be useful to map a particular skin chromophore. For instance, Diebele et al. ${ }^{10}$ and Bekina et al. ${ }^{5}$ proposed the following parameters to account for bilirubin $(B)$ and erythema $(E)$, respectively:

$B=I_{450}(i, j) / I_{660}(i, j)$,

$E=I_{660}(i, j) / I_{545}(i, j)$,

where $I_{\lambda}$ is the intensity of diffuse light reflected from the skin at a specific wavelength of a multispectral system composed of four different spectral bands-three in the visible (450, 545, and $660 \mathrm{~nm})$ and one in the infrared ranges $(940 \mathrm{~nm})$.

The same authors also proposed a melanoma index $(p)$ as follows:

$p=k \cdot\left\{I_{540}(i, j) /\left[I_{650}(i, j) * I_{950}(i, j)\right]\right\}$,

where $k$ is the intensity coefficient that describes the white etalon reference used for the calibration of the system.

In particular, the eight wavelengths available in our system without interpolation were used to compute the former parameters. To resemble those proposed by other authors, the closest available wavelengths were chosen.

Similarly, Emery et al. ${ }^{3}$ suggested that the SIAscope commercial system could contribute to the management of pigmented skin lesions. SIAscope maps the dermal melanin of lesions, and melanomas usually have more dermal melanin than other skin lesions. Specifically, the system also captured four different images ${ }^{17}$ (three in the RGB channels and one in the infrared), and, to avoid calibration, the ratio images of green over red and blue over red were calculated pixel-bypixel to account for the concentrations of blood and melanin, with the help of a conversion table.

Once all images including parameters from the three groups were obtained, a mask was created to segment the lesion from the skin. The segmentation algorithm used was based on the Otsu method ${ }^{18}$ and consisted of maximizing the between-class 
variance of the lesion and the skin pixel values to define a threshold based on the intensity of the histogram at the bluest reflectance image $(414 \mathrm{~nm})$. This image was used as it provided information from the most superficial layer of the skin, allowing for a better discrimination between the lesion and the surrounding healthy skin. Furthermore, for those lesions that were not homogeneous, the reflectance image was divided into four different subimages, allowing different thresholds adapted to the different areas of the lesion to be calculated.

Next, statistical descriptors were obtained for every segmented lesion for all parameters, i.e., mean $(\tilde{x})$, standard deviation $(\sigma)$, maximum, and minimum. As a first approach to the extraction of textural information, we used the analysis of the statistical properties of the histogram for any of the parameters calculated, also known as first-order statistics. ${ }^{19,20}$ This analysis includes the study of some features such as entropy $(E p)$, a well-known statistical measure of randomness, energy $(E n)$, a numerical descriptor of the image uniformity having 1 as its maximum value for a constant image, and the third central moment $\left(\mu_{3}\right)$, which accounts for the skewness of the histogram. The mathematical descriptions of these features are:

$$
\begin{aligned}
& E p=-\sum_{i=0}^{n-1} P_{i} \log _{2}\left(P_{i}\right), \\
& E n=\sum_{i=0}^{n-1} P_{i}^{2}, \\
& \mu_{3}=-\sum_{i=0}^{n-1}(i-m)^{3} P_{i},
\end{aligned}
$$

where $n$ is the number of bins or intervals into which the histogram is divided, $P_{i}$ is the relative frequency of the bin $i$ of the histogram, and $m$ is the mean of the parameter.

\subsection{Classification Algorithm}

A classification algorithm was developed to decide which lesions were malignant (melanomas and basal cell carcinomas). To this end, the data were split into a training set and a validation set of the same size including half of the nevi, basal cell carcinomas, and melanomas. Using the training set, upper and lower thresholds were first defined for each parameter as the interval limits that included all nevi. The upper (lower) threshold was chosen as the value of the nevus with the highest (lowest) value after some nevi were discarded according to

Nevi outliers $=\tilde{x}_{\text {nevi }} \pm 2 \cdot \sigma_{\text {nevi }}$,

where $\tilde{x}_{\text {nevi }}$ and $\sigma_{\text {nevi }}$ are the average and standard deviation, respectively, in terms of each parameter calculated from all segmented nevi lesions in the training set. For instance, $\tilde{x}_{\text {nevi }}$ and $\sigma_{\text {nevi }}$ can be those corresponding to the energy of the reflectance at $995 \mathrm{~nm}$ or to the maximum lightness $(L *)$ of all pixels belonging to a lesion diagnosed as nevus.

All lesions above or under the upper and lower thresholds for at least one parameter were classified as malignant.

The classification algorithm worked as follows: after calculating the thresholds of all parameters, they were ordered according to the number of malignant lesions they allowed classifying. Accordingly, the first parameter on the list was that allowing the greatest number of malignant lesions in the training set to be classified, the second one was that allowing the second greatest number, and so forth. The algorithm then started from the first of the list alone and calculated the corresponding sensitivity, i.e., the percentage of malignant lesions classified as such. The second parameter of the list was then chosen to perform the classification together with the first one, and the sensitivity was computed again. If the second parameter did not allow improving the classification with at least one more malignant lesion detected, it was discarded as it was considered to be redundant. Otherwise, it was included. Next, the third parameter on the list was added to the first two and the sensitivity was calculated again, repeating the described process until the addition of more parameters did not improve the sensitivity of malignant lesions of the training set. The sensitivity and specificity values achieved with the formerly chosen parameters were then calculated for the validation set exclusively.

Finally, the same analysis was carried out again but without including basal cell carcinomas in the training and validation sets to be able to compare the obtained results in terms of sensitivity and specificity with those from other studies that only tested melanomas and nevi and to not artificially inflate the system accuracy.

\section{Results}

The developed system was used to analyze 564 pigmented and nonpigmented skin lesions at the Hospital Clínic i Provincial de Barcelona (Barcelona, Spain) and the Università di Modena e Reggio Emilia (Modena, Italy). All patients provided written informed consent before any examination and ethical committee approval was obtained. The study complied with the tenets of the 1975 Declaration of Helsinki (Tokyo revision, 2004). The lesions were diagnosed by dermatologists (SP and JM in Barcelona, GP and SB in Modena) using a commercial dermoscope and the confocal laser scanning microscope VivaScope ${ }^{\circledR}$ 1500 from MAVIG. When malignancy was suspected, the lesion was excised and a histological analysis was carried out.

From the 564 lesions measured, 11\% could not be properly segmented: $4.4 \%$ had low pigmentation, $3.2 \%$ had no welldefined borders, $1.6 \%$ were bigger than the field of view of the system, $0.9 \%$ presented misalignments along the spectral images taken at different wavelengths due to the patient's breath movement, $0.6 \%$ presented artifacts such as hairs, and $0.3 \%$ many different colors making the segmentation difficult.

From the remaining 502 lesions that could be segmented, 73 were excluded as they were diagnosed as seborrheic keratosis, squamous carcinomas, and other benign lesions such as angiomas or dermatofibromas. However, there were not enough samples in each category to be included in the study.

Finally, 429 skin lesions were included in the analysis: 290 were benign lesions classified as nevi (melanocytic, dysplastic, blue, junctional, and Spitz nevi) and 139 were malignant (95 melanomas and 44 basal cell carcinomas). It is also worth noting that the limitation of most studies is the inclusion of preselected lesions according to the histopathological diagnosis whereas our study was a prospective study including consecutive lesions that could enter in the differential diagnosis of melanoma. In general, some basal cell carcinomas can be easily diagnosed clinically but dangerous melanomas, such as amelanotic melanoma or nodular melanoma, can be easily misdiagnosed as basal cell carcinomas. Therefore, the approach used in our study is more 
approximate to what happens in real life, where basal cell carcinomas are at least 10 times more frequent than melanomas and can be misdiagnosed if not considered.

Figure 3 shows representative VIS-NIR reflectance images of a nevus, a melanoma, and a basal cell carcinoma. The figure shows that nevi are usually more homogeneous at all wavelengths. Furthermore, the infrared light, which penetrates deeper in the skin, shows that melanomas generally grow deeper.

The averaged reflectance ( $\pm \sigma$, standard deviation) of nevi, melanomas, and basal cell carcinomas can be seen in Fig. 4 . In agreement with previous publications, ${ }^{11,12}$ the averaged reflectance of melanomas (or equivalently absorbance) is generally lower (higher) than that of nevi. However, this difference decreases when considering the great variance among lesions of the same type, which makes it difficult to classify them if only averaged values are taken into account.

To overcome this limitation, histograms for the whole lesion with all parameters were also plotted. Figure 5 shows specific examples of histograms of a nevus and a melanoma in terms of reflectance at 415 and $675 \mathrm{~nm}$. The averaged spectral reflectance $(\tilde{x})$, the standard deviation $(\sigma)$, maximum, minimum, and corresponding $E p, E n$, and $\mu_{3}$ are also shown.

The results show that the averaged reflectance, the standard deviation, and the maximum and minimum at $415 \mathrm{~nm}$ for both lesions are very similar. In contrast, the histograms look completely different; the nevus is linked to a higher $E n$ and a lower $E p$ since its pigmentation is more uniform than the melanoma. With regard to the skewness $\left(\mu_{3}\right)$, the nevus presents a practically symmetrical distribution and, thus, a lower value than the melanoma. On the other hand, both lesions have enhanced spectral differences at $675 \mathrm{~nm}$, as expected from Fig. 4, and, thus, the averaged reflectance is clearly different; however, the $E n$ and the $E p$ remain more similar in this case in comparison with $415 \mathrm{~nm}$, whereas the melanoma is skewed to the left (negative) in terms of the third central moment, contrary to what was obtained for the nevus.

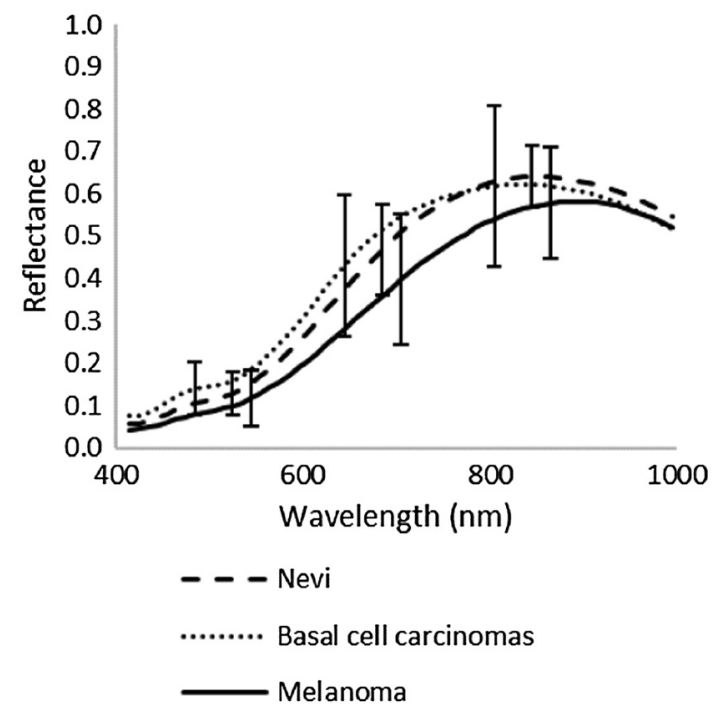

Fig. 4 Averaged reflectance ( $\pm \sigma$, standard deviation) of nevi, melanomas, and basal cell carcinomas.

In summary, we might conclude that the texture information might allow the differentiation between nevi and malignant lesions (melanomas and basal cell carcinomas).

From all the parameters calculated, only 15 were not redundant and finally selected for use in the classification algorithm. As formerly described, only those that detected a larger number of malignant lesions were chosen, until the addition of more parameters did not improve the results in terms of sensitivity in the training set, i.e., the detection of malignant lesions.

- Eight were spectral reflectance and absorbance of the lesion (first group): $E n$ of $\mathrm{Abs}_{875}, \mu_{3}$ of $\operatorname{Refl}_{745}, \tilde{x}$ of $\operatorname{Refl}_{645}$, and $\sigma$ of $\mathrm{Abs}_{935}$. Also, differences between lesion and surrounding skin in terms $E p$ of $\mathrm{Abs}_{465}, E n$ of $\mathrm{Abs}_{635}, \mu_{3}$ of $\mathrm{Abs}_{985}$, and $\mu_{3}$ of $\operatorname{Refl}_{975}$.

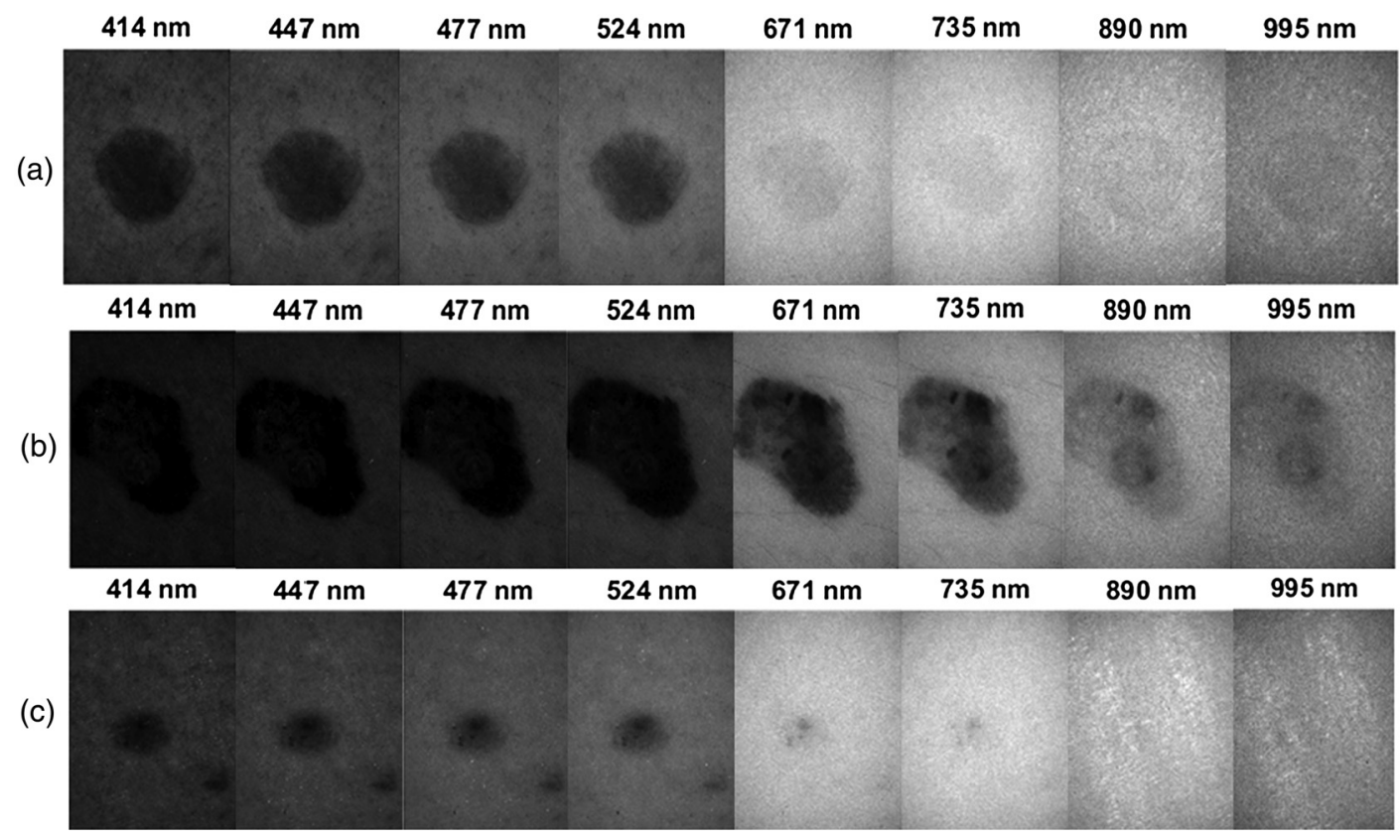

Fig. 3 Reflectance images obtained with the system at different wavelengths analyzed: (a) nevus, (b) melanoma, and (c) basal cell carcinoma. 


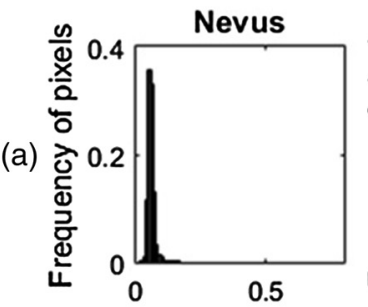

Reflectance $415 \mathrm{~nm}$ $\tilde{x}=0.06$

$\sigma=0.01$

Maximum $=0.18$

Minimum $=0.01$

$E p=2.21$

$E n=0.27$

$\mu_{3}=2.44 \mathrm{e}-6$

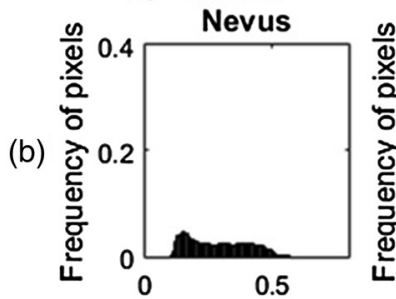

Reflectance $675 \mathrm{~nm}$

$\tilde{x}=0.28$

$\sigma=0.12$

Maximum $=0.57$

Minimum $=0.09$

$E p=5.30$

$E n=0.03$ $\mu_{3}=3.88 \mathrm{e}-4$

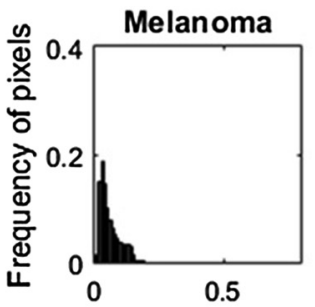

Reflectance $415 \mathrm{~nm}$

$\tilde{x}=0.06$

$\sigma=0.01$

Maximum $=0.20$

Minimum $=0.01$

$E p=3.51$

$E n=0.11$

$\mu_{3}=4.34 \mathrm{e}-5$

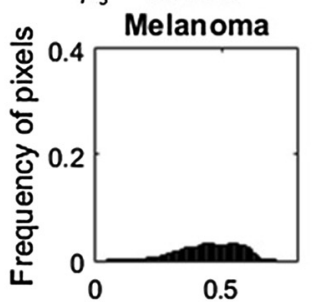

Reflectance $675 \mathrm{~nm}$ $\tilde{x}=0.46$

$\sigma=0.11$

Maximum $=0.73$

Minimum $=0.05$

$E p=5.47$

$E n=0.02$

$\mu_{3}=-6.76 \mathrm{e}-4$
Fig. 5 Histograms of (a) a nevus and a melanoma in terms of spectral reflectance at $415 \mathrm{~nm}$ and (b) at $675 \mathrm{~nm}$, with their respective values of mean $(\tilde{x})$, standard deviation $(\sigma)$, maximum, minimum, entropy $(E p)$, energy $(E n)$, and third central moment $\left(\mu_{3}\right)$.
- Four were color-based parameters (second group): maximum of $L *, \sigma$ of $a *$ as CIELAB colorimetric coordinates, and minimum $\Delta E$ with the surrounding healthy skin as the reference, and maximum ITA.

- Three were empirical parameters (third group): En of Emp1, maximum of Emp2, maximum of Emp3, which are defined as follows:

$$
\begin{aligned}
& \text { Emp1 }=\log \left\{\operatorname{Refl}_{524}(i, j) /\left[\operatorname{Refl}_{671}(i, j) \cdot \operatorname{Refl}_{995}(i, j)\right]\right\}, \\
& \text { Emp2 }=\operatorname{Refl}_{671}^{2}(i, j) / \operatorname{Refl}_{524}^{2}(i, j), \\
& \text { Emp3 }=\frac{\operatorname{Refl}_{671}(i, j)}{\operatorname{Refl}_{995}(i, j)} .
\end{aligned}
$$

The following scatter plots (Fig. 6) show three specific examples from the three groups of selected parameters. Since the number of melanomas finally included in the study was odd, one extra melanoma was considered in the validation set.

The corresponding upper and lower thresholds used for classification were calculated with the training set while the samples shown correspond to the validation set. The plots show that some of the melanomas and basal cell carcinomas tend to have values beyond the upper/lower thresholds.

With the classification algorithm based on these 15 parameters, 6 out of 47 melanomas and none of the 22 basal cell carcinomas were misclassified (91.3\% sensitivity); in contrast, 66 nevi from 145 were classified as malignant (54.5\% specificity). Here, sensitivity or true positive rate is the probability of detecting malignant lesions (melanomas and basal cell carcinomas) and the specificity or true negative rate is the proportion of nevi correctly identified.

As commented above, the analysis was repeated but only including nevi and melanomas in the training and validation sets. In this case, the sensitivity and specificity of the system were of $87.2 \%$ and $54.5 \%$, respectively.

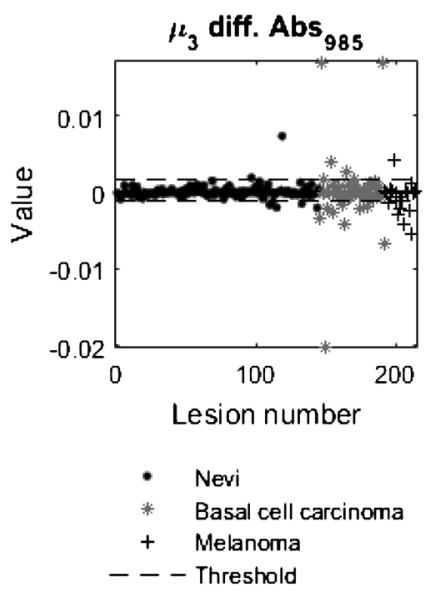

(a)

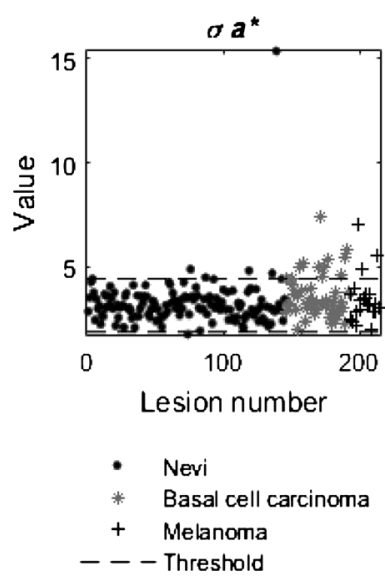

(b)

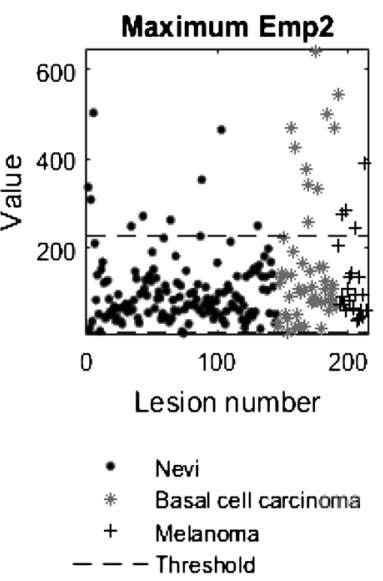

(c)

Fig. 6 Scatter plots from three specific parameters: (a) differences between the lesion and the skin in terms of $\mu_{3}$ of Refl ${ }_{985}$, (b) $\sigma$ of $a *$ of the CIELAB color space, and (c) maximum of Emp2. The thresholds shown are those calculated with the training set while the samples correspond to those of the validation set. 


\section{Discussion and Conclusions}

In a study of the first SIAscope scoring system applied to a dataset of 348 pigmented lesions (52 melanomas and the remainder nonmelanoma lesions, mostly nevi), Moncrieff et al. ${ }^{21}$ obtained sensitivity and specificity values of $82.7 \%$ and $80.1 \%$, respectively. The SIAscope is a multispectral system with four narrowbands (from 400 to $1000 \mathrm{~nm}$ ) that provides information about the amount of collagen, hemoglobin, and melanin distribution in the epidermis and dermis of pigmented skin lesions in the form of maps called SIAscans. These maps, presented by the MoleMate software, have to be interpreted by general practitioners or dermatologists who have previously received training. $^{22}$

Similarly, Haniffa et al. ${ }^{23}$ conducted a study that included 881 pigmented lesions. The observations, carried out by a 3year-experienced dermatologist using the latest SIAscope software, obtained $87 \%$ and $91 \%$ sensitivity and specificity, respectively. In the same study, the observations made by a dermatologist with 20 years of experience resulted in $94 \%$ sensitivity and $91 \%$ specificity. The authors concluded that the use of SIAscope by an experienced dermatologist made no additional contribution over clinical diagnosis.

In a more recent study that analyzed 188 lesions including three types of malignant lesions ( 21 melanomas, 9 basal cell carcinomas, and 5 squamous cell carcinomas) and various benign lesions (122 nevi, 23 seborrheic keratosis, 7 dermatofibromas, and 1 cherry angioma), Sgouros et al. ${ }^{24}$ concluded that, although SIAscope was not superior, it could support the results of dermoscopy. In this study, the sensitivity and specificity for suspected malignant lesions were $85.7 \%$ and $65.4 \%$, respectively. The lower specificity compared with previous studies results from the inclusion of more types of malignant lesions. The authors concluded that SIAscope should not be considered a replacement for the standard diagnostic procedure, but an additional tool for nondermatologist clinicians.

Taking into account that the classification algorithm proposed in this article does not require the skills of a dermatologist to interpret a map, our study provides slightly higher sensitivity (91.3\%) and lower specificity $(54.5 \%$ ) values than previous studies. Consequently, the inclusion of texture information can be considered relevant for the detection of melanomas and basal cell carcinomas.

However, one should bear in mind that the sensitivity decreases to $87.2 \%$ when basal cell carcinomas are not considered. Nevertheless, this value is still similar to that obtained by an experienced dermatologist using the SIAscope.

On the other hand, MelaFind ${ }^{\circledR}$ is a 10 -wavelenghts $(430,470$, $500,550,600,650,700,770,880$, and $950 \mathrm{~nm}$ ) system that generates six scores based on constrained linear classifiers for each measured lesion. In MelaFind ${ }^{\circledR}$, each classifier is trained to differentiate melanomas from other pigmented lesions such as common nevi, low-grade dysplastic nevi, congenital nevi, seborrheic keratosis, solar lentigos, and pigmented basal cell carcinomas from a database of $\sim 10,000$ excised lesions. ${ }^{25}$ A lesion is recommended for biopsy if all six scores are above the threshold value. ${ }^{22}$ After the measurement, the system provides a disorganization value and a treatment suggestion for dermatologists: positive or negative for high degree of morphological disorganization lesion, where positive means the lesion should be considered for biopsy.

The FDA Summary of Safety and Effectiveness Data of MelaFind ${ }^{\circledR 26}$ shows a much higher sensitivity (98.3\%) to detect in situ and invasive melanomas (172/175 melanomas detected) than the SIAScope; however, the specificity was only of $10.8 \%$ (157/1457 of high grade dysplastic nevi, atypical melanocytic proliferation/hyperplasia lesions were classified as melanomas). Therefore, the use of MelaFind ${ }^{\circledR}$ generates a large number of false positives and, consequently, a large number of unnecessary biopsies. Other investigations in which different dermatologists were asked to evaluate and diagnose a specific number of lesions with and without information from the MelaFind ${ }^{\circledR}$ were carried out. The averaged sensitivities and specificities obtained were very similar to those previously shown, ranging from $96.9 \%$ to $98.3 \%$ and from $9.2 \%$ to $9.9 \%,{ }^{4,27}$ respectively. The authors concluded that the information obtained with the MelaFind ${ }^{\circledR}$ should be used to decide the need for a biopsy, since in case of an experienced dermatologist MelaFind ${ }^{\circledR}$ could improve biopsy sensitivity with a modest effect on biopsy specificity.

The lower sensitivity shown in our system could be improved by defining more restricted upper and lower thresholds. However, this would also result in an increase of false positives and, thus, a marked reduction of the specificity, which is unacceptable from the dermatologists' point of view. In fact, the detection of malignant lesions at early stages, when they can still be controlled and successfully excised, is crucial when dealing with skin cancer, and this is the reason why dermatologists are more concerned with increasing sensitivity than specificity.

On the other hand, we should underscore that, in contrast with MelaFind ${ }^{\circledR}$, our study only compared melanomas and basal cell carcinomas with different kinds of nevi (common, melanocytic, dysplastic, blue, junctional, and Spitz nevi) and melanomas with nevi.

Using Eq. (6) described in Sec. 2.1, Diebele et al. ${ }^{10}$ found values of $94 \%$ and $89 \%$ for sensitivity and specificity, respectively. In this case, the system developed by the authors consisted of a multispectral imaging camera (Nuance EX) that contained a CCD imaging sensor, a solid-state liquid crystal filter with a polarizer, a wavelength tuning element (from 450 to $950 \mathrm{~nm}$ in steps of $10 \mathrm{~nm}$ ), a spectral optimized lens, and internal optics. The illumination system was a ring of halogen lamps with a polarizer orthogonal to the camera to remove the artifacts caused by light reflection. However, they only analyzed 65 nevi and 17 melanomas and a further verification of the algorithm is still pending. Our system obtained a slightly lower sensitivity when excluding basal cell carcinomas, taking into account that in contrast with the abovementioned study, it also included dysplastic nevi and nonpigmented lesions. These lesions present a further difficulty in the discrimination of melanomas, since they are unusual benign moles that may resemble melanomas. In addition, our study included many more lesions.

On the other hand, artificial intelligence is starting to enter in the medicine field. ${ }^{28}$ Specifically, Esteva et al. ${ }^{29}$ used a deep convolutional neural network (CNN) to classify skin lesions from dermoscopic images. The algorithm was trained from a dataset of more than 100,000 images from 18 different cliniciancurated, open-access online repositories and from the Stanford University Medical Center. An area under curve of $96 \%$ for the detection of carcinomas and $94 \%$ for the detection of melanomas was obtained, improving the performance of most of the 21 dermatologist that were asked to classify hundreds of lesions from a dermoscopic image. The conclusion of the study was that CNN could be a powerful tool for huge image datasets, and encouraging results will probably be obtained in the coming years. However, this is not the best tool for pilot studies in 
which new technologies are tested and the database of lesions is small.

In conclusion, the addition of textural information, which to our knowledge has not been yet considered in any study, was shown to be more useful for the diagnosis of malignant lesions (melanomas and basal cell carcinomas) than the sole use of averaged spectral and color information. The sensitivity (91.3\%) and specificity $(54.5 \%)$ values slightly improved in some cases over those previously achieved by means of other multispectral systems without the need for an experienced dermatologist as it was based on a complete automatic algorithm. However, when only the melanomas and nevi were considered, the sensitivity decreased to $87.2 \%$, although this value was still similar to that obtained by experienced dermatologists through averaged spectral and color features.

The system proposed has a specificity similar to that of confocal microscopy $(55.1 \%)^{30}$ but is much closer to dermoscopy $(84.1 \%)^{31}$ than Melafind ${ }^{\circledR}(10.8 \%)$. On the other hand, the system has a slightly lower sensitivity than Melafind ${ }^{\circledR}(98.3 \%)$ and confocal microscopy $(96.3 \%)^{29}$ but is closer than dermoscopy $(89.2 \%){ }^{31}$

Consequently, our system can help to improve the diagnosis of skin cancer as a supporting tool to dermoscopy and confocal microscopy. In fact, the combination of different technologies might be crucial to improving sensitivity and specificity in this field.

\section{Disclosures}

No conflicts of interest, financial or otherwise, are declared by the authors.

\section{Acknowledgments}

This work was supported by the European Union through the project DIAGNOPTICS “diagnosis of skin cancer using optics" (ICT PSP seventh call for proposals 2013).

\section{References}

1. W. Klaus and J. R. Allen, Fitzpatrick's Color Atlas and Synopsis of Clinical Dermatology, McGraw-Hill Professional, New York (2009).

2. G. Guy et al., "Melanoma treatment costs," Am. J. Prev. Med. 43(5), 537-545 (2012).

3. J. D. Emery et al., "Accuracy of SIAscopy for pigmented skin lesions encountered in primary care: development and validation of a new diagnostic algorithm," BMC Dermatol. 10(9), 1-9 (2010).

4. G. Monheit et al., "The performance of MelaFind," Arch. Dermatol. 147(2), 188-194 (2011).

5. A. Bekina et al., "Multispectral assessment of skin malformations using a modified video-microscope," Latv. J. Phys. Tech. Sci. 49(5), 4-8 (2012).

6. D. Kapsokalyvas et al., "Spectral morphological analysis of skin lesions with a polarization multispectral dermoscope," Opt. Express 21(4), 4826-4840 (2013).

7. M. Ares et al., "Handheld 3D scanning system for in-vivo imaging of skin cancer," in 5th Int. Conf. on 3D Body Scanning Technologies, pp. 231-236, Lugano, Switzerland(2014).

8. S. K. Özdemir et al., "Noninvasive blood flow measurement using speckle signals from a self-mixing laser diode: in vitro and in vivo experiments," Opt. Eng. 39(9), 2574-2580 (2000).

9. G. Pellacani et al., "The impact of in vivo reflectance confocal microscopy for the diagnostic accuracy of melanoma and equivocal melanocytic lesions," J. Invest. Dermatol. 127(12), 2759-2765 (2007).

10. I. Diebele et al., "Clinical evaluation of melanomas and common nevi by spectral imaging," Biomed. Opt. Express 3(3), 467-472 (2012).
11. B. Murphy et al., "Toward the discrimination of early melanoma from common and dysplastic nevus using fiber optic diffuse reflectance spectroscopy," J. Biomed. Opt. 10(6), 064020 (2005).

12. I. Kuzmina et al., "Towards noncontact skin melanoma selection by multispectral imaging analysis," J. Biomed. Opt. 16(6), 060502 (2011).

13. CIE Publication 142, "Improvement to Industrial Colour-Difference Evaluation," Commission Internationale de L'Éclairage, Vienna, Austria (2001).

14. J. Pladellorens et al., "A device for the color measurement and detection of spots on the skin," Skin Res. Technol. 14, 65-70 (2008).

15. I. L. Weatherall and B. D. Coombs, "Skin color measurements in terms of CIELAB color space values," J. Invest. Dermatol. 99(4), 468-473 (1992).

16. ASTM E2214-08, Standard Practice for Specifying and Verifying the Performance of Color-Measuring Instruments, American Society for Testing and Materials, West Conshohocken, Pennsylvania (2008).

17. S. Preece et al., "Method and apparatus for detecting the presence of dermal melanin in epithelial tissue," U.S. Patent No. 20070161910A1 (2007).

18. D. Liu and J. Yu, "Otsu method and K-means," in Proc. 9th Int. Conf. on Hybrid Intelligent Systems (HIS 2009), Vol. 1, pp. 344-349 (2009).

19. R. C. Gonzalez, R. E. Woods, and S. L. Eddins, Digital Image Processing Using Matlab, Prentice Hall, Upper Saddle River, New Jersey (2004).

20. G. X. Ritter and J. N. Wilson, Handbook of Computer Vision Algorithms in Image Algebra, CRC Press, Boca Raton (2001).

21. M. Moncrieff et al., "Spectrophotometric intracutaneous analysis: a new technique for imaging pigmented skin lesions," $\mathrm{Br}$. J. Dermatol. 146, 448-457 (2002).

22. A. Gadeleya, M. D. Goodson, and D. Grossman, "Strategies for early melanoma detection: approaches to the patient with nevi," J. Am. Acad. Dermatol. 60(5), 719-735 (2009).

23. M. A. Haniffa, J. J. Lloyd, and C. M. Lawrence, "The use of a spectrophotometric intracutaneous analysis device in the real-time diagnosis of melanoma in the setting of a melanoma screening clinic," $B r . J$. Dermatol. 156(6), 1350-1352 (2007).

24. D. Sgouros et al., "Assessment of SIAscopy in the triage of suspicious skin tumours," Skin Res. Technol. 20, 440-444 (2014).

25. A. Hauschild et al., "To execise or not: impact of MelaFind on German dermatologists' decisions to biopsy atypical lesions," J. Dtsch. Dermatol. 12(12), 606-614 (2014).

26. “MelaFind ${ }^{\circledR}$ PMA P090012-package insert," MELA Sciences (2012).

27. M. Burroni et al., "Dysplastic naevus vs. in situ melanoma: digital dermoscopy analysis," Br. J. Dermatol. 152(4), 679-684 (2005).

28. Q. Li et al., "Medical image classification with convolutional neural network," in 13th Int. Conf. on Control, Automation Robotics and Vision, Th25.2, pp. 844-848, Singapore (2014).

29. A. Esteva et al., "Dermatologist-level classification of skin cancer with deep neural networks," Nature 542(7639), 115-118 (2017).

30. G. Pellacani et al., "Reflectance confocal microscopy for the diagnostic accuracy of melanoma and equivocal melanocytic lesions," J. Invest. Dermatol. 127(12), 2759-2765 (2007).

31. R. Langley et al., "The diagnosis accuracy of in vivo confocal scanning laser microscopy compared to dermoscopy of benign and malignant melanocytic lesions: a prospective study," Dermatology 215, 365-372 (2007).

Xana Delpueyo received her BSc degree in optics and optometry in 2011 and her MSc in optometry and vision sciences in 2013 at the Technical University of Catalonia (UPC). She is currently a predoctoral researcher at the Centre for Sensors, Instruments and Systems Development (CD6) of the UPC where she enrolled the doctorate program in optical engineering. Her main research interests are multispectral and hyperspectral imaging systems and colour measurement.

Meritxell Vilaseca received her BSc degree in physics at the Autonomous University of Barcelona in 2000 and a PhD in optical engineering from the UPC in 2005. She completed her degree in optics and optometry at the UPC in 1996. She is currently an associate professor at the UPC and a researcher at the Centre for Sensors, Instruments and Systems Development (CD6). Her work focuses on optical engineering. 
Santiago Royo received his $\mathrm{PhD}$ in applied optics from the Universitat Politècnica de Catalunya (UPC-BarcelonaTech) in 1999. He is currently an associate professor at UPC and director of the Center for Sensor, Instruments and System Development (CD6). He has participated and led research projects in optical metrology and engineering, leading him to publish over 50 full-text publications and 10 patents. $\mathrm{He}$ is cofounder of two spin-off companies: SnellOptics Spain in 2002, and ObsTech SpA Santiago, Chile in 2012.

Miguel Ares received his $\mathrm{PhD}$ in optical engineering in 2009 from the UPC. He is currently a postdoctoral research fellow in the CD6 - UPC, leading the research line of 3-D human body scanning, which is focused in the development of technologies and methods based on optical and computer vision techniques. He has participated in 13 funded research projects and have published 4 articles and 1 patent.

Laura Rey-Barroso received his MSc degree in biomedical engineering from the University Carlos III of Madrid, where she made some research about the new applications of spectroscopy. Her interest in photonic sciences made her join the master in photonics of the Polytechnic University of Catalonia where she specializes in biophotonics and nanophotonics. In 2016, she joins UPC to investigate about skin cancer diagnosis by multiespectral techniques, like infrared imaging spectroscopy.

Ferran Sanabria received his MSc degree in computer science and degree in informatics engineering from the Universitat Politècnica de Catalunya (UPC) in 1992 and his PhD in optical engineering in 2015. In 1997, he joined the Centre for Sensors, Instruments \& Systems Development (CD6-UPC) where he participated in several publicly funded projects and partnership contracts with companies. He is currently working at (CD6-UPC) as computer engineer and he is an associate professor in Automatic Control Department (ESAlI-UPC).

Susana Puig is a dermatologist, the director of the Dermatology Service at the University Hospital Clinic of Barcelona, Barcelona, Spain and the director of the research program melanoma: imaging, genetics and immunology at the IDIBAPS, Barcelona, Spain. Special research areas are dermoscopy, digital follow-up of melanocytic tumors, confocal microscopy and genetics of melanoma with the publication of more than 300 scientific articles, 2 dermoscopy books and more than 30 book chapters.

Josep Malvehy is a consultant dermatologist and director of the Melanoma Unit of the Hospital Clinic of Barcelona. He is a professor at the University of Barcelona. His main field of expertise is noninvasive skin diagnosis and treatment of malignant neoplasms of the skin (melanoma and nonmelanoma skin cancer) with biophotonics and other methods. He is an investigator of the IDIBAPS (Institut d'Investigació August Pi i Sunyer).

Giovanni Pellacani: Biography is not available.

Fernando Noguero received his technical engineering in industrial electronics from the Technical University of Catalonia (UPC) in 1980. He has been working on developing and setting in motion process automation projects for big factories. He is currently working as quality manager in a Medical Devices Company. His main tasks are the implementation and maintenance of the ISO 13485 Standard, the technical file of the company products and the different quality control points of the products.

Giuseppe Solomita has been working in the field of international sales starting in 1998 (sales manager - Sanyo Electric Ltd., Osaka) and moved on to Mavig GmbH, Munich in 2007. As the head of the VivaScope Systems Department, he has built up a strong relationship with over 200 academic centres all over Europe, and has increased the adoption of the Vivascope Technology in research and clinical applications through accredited ingenious training programs.

Thierry Bosch is currently a professor with ENSEEIHT-INPT, Toulouse, France, and the head of the Optoelectronics for Embedded Systems Research Group with LAAS-CNRS, Toulouse. He is specialized in optical feedback interferometry for laser-based sensing applications. His current research interests include laser industrial instrumentation development, including range finding techniques, vibration and velocity measurements for mechatronics, quality control, non destructive testing, transport and biosensing. 